\title{
FeII, FeIII and MgII spectra of Be stars
}

\author{
L. H. Barrera and J. Dachs \\ Astronomisches Institut, Ruhr-Universität, Postfach 102148, D-44780 Bochum, Germany.
}

and

T. W. Berghöfer

Max-Planck-Institut für Extraterrestrische Physik, Giessenbachstraße, D-85748 Garching, Germany.

For a total of 33 bright Be stars, archival high-dispersion spectrograms obtained by the International Ultraviolet Explorer (IUE) satellite were analyzed together with optical spectrograms in order to study the FeII, FeIII and MgII spectra in the entire $1600 \AA$ to $6500 \AA$ wavelength range. Ultraviolet FeII resonance lines are always fourd to be in absorption which usually is of interstellar origin. $\mathrm{B}_{\mathrm{y}}$ applying curve-of-growth methods to equivalent widths measured for FelI multiplets UV-1 through UV-8, column densities of interstellar FeII are derived ranging typically between about $10^{14}$ and $10^{15} \mathrm{~cm}^{-2}$. For a few program stars showing shell-type spectra in the optical region, additional FeII resonance absorption lines are detected starting from excited fine-structure levels above the ground state, pointing to FeII absorption occurring in the dense circumstellar envelope in front of these star. Analysis of their equivalent widths is used to infer typical column densities of circumstellar ground state FeII ions of order $10^{15} \mathrm{~cm}^{-2}$.

FeII emission lines are only observed in the optical part of Be star spectra, involving transitions connecting both quartet and sextet levels with minimum excitation energies of about $2.5 \mathrm{eV}$. The relatively large strengths of optical FeII emission lines (with equivalent widths up to $0.7 \AA$ ) are compared to upper limits for undetected FeII resonance line emission in the circumstellar disks. This leads to the conclusion that FeII emission in Be star envelopes is mainly caused by radiative recombination of FeIII. This conclusion is also supported by the fact that in variable Be star spectra, equivalent widths of FeII emission lines are correlated with optical depths for electron scattering as inferred from variable extensions of $\mathrm{H} \alpha$ emission line wings. For about one quarter of program stars unusually strong FeIII absorption is observed in front of the stars in multiplets UV-34 and UV-48, arising from metastable lower levels about $5 \mathrm{eV}$ above the FeIII ground state. These stars either show strong CIV stellar wind characteristics in their ultraviolet spectra (HR 2855 , HR 4140 , HR 5440, HR 8539), or shell-type features in their optical spectra (HR 5941, HR 8260, HR 8402).

MgII optical (multiplet 4) and ultraviolet (UV-1) lines contain photospheric (absorption) as well as circumstellar (emission or shell absorption) components or both and for multiplet UV-1 also strong interstellar absorption. Intensities of MgII emission are correlated to intensities of FelI emission lines. Four different cases of the combination of photospheric, interstellar and variable circumstellar 
absorption and emission features of .IgII UV-1 are illustrated in Fig. 1 (HR 3498, HR 4140, HR 5440 and HR 6118). Partly, our conclusions are supported by data collected in the Be Star Atlas of High-Resolution Spectra by Doazan et al. (ESA SP-1147, 1991).

This work has been supported by a grant from the Deutscher Akademischer Austauschdienst (DAAD) to L.H. Barrera.
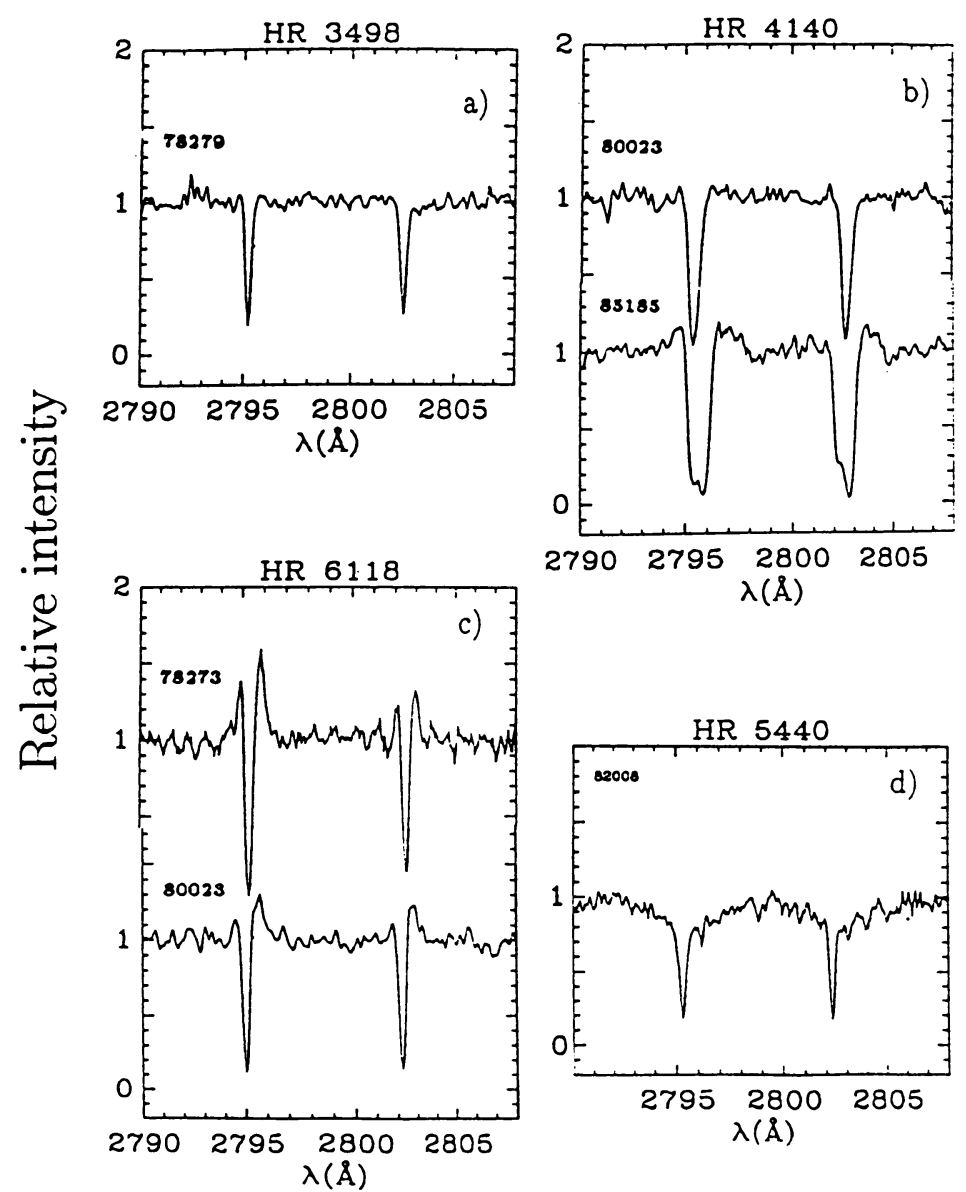

Fig. 1. Resonance lines of MgII LV-1, $\lambda 2795$ and $\lambda 2802$; a) interstellar absorption; b) interstellar absorption superimposed by variable circumstellar absorption and emission; c) variable circumstellar emission superimposed by interstellar absorption; d) photospheric absorption superimposed by interstellar absorption. Dates are indicated as (year - 1900)*1000 + day number. 